\title{
An Improvement of Network Life Time using DLQAR Protocol in Wireless Sensor Network
}

\author{
Kulvir Singh \\ Lecturer \\ Deptarment of CSE \\ GPC GTBGarh, Moga
}

\author{
Sunny Behal \\ Assistant Professor, \\ Department of CSE, \\ SBSSTC, Ferozepur
}

\author{
Vishal Kumar Arora \\ Assistant Professor, \\ Department of CSE, \\ SBSSTC, Ferozepur
}

\begin{abstract}
A Wireless Sensor Network(WSN) is critical network defined with restricted resources and constraints. Wireless Sensor Network are used multiple application like security ,military and health application.To optimize the network route and network life, under these constraints is always a challenge. In this paper, a multi parameter based hop selection analysis based algorithm is proposed to generate the optimized route over the sensor network based on Residual energy ,Failure rate and sensing range using DLQAR protocol. The no. of alive node, dead node, residual energy, energy consumption terms are used to analyze the proposed algorithm. These parameter dynamic analyze the network route and change the network route as per requirements. The proposed work uses the threshold value to perform the critical node elimination. The results obtained show that the proposed algorithm is better as compared to existing algorithm in terms of alive node, dead node, residual energy, energy consumption.
\end{abstract}

\section{Keywords}

WSN, Routing, Constraints, Failure Probability .

\section{INTRODUCTION}

Wireless Sensor Network(WSN) is a wireless network consisting of spatially autonomous devices using sensors to cooperatively monitor physically or environmental conditions, such as temperature, sound, vibration, motion or pollutant, at different locations[2]. Wireless Sensor Network is one of the real time network defined under various restrictions and specification. These specifications include network level constraints, node level constraints and environment level constraints. A senor network is composed using large number of tinny devices called sensor nodes placed in small network area. Sensor nodes are randomly deployed in remote location where human intervention is difficult or sometimes almost impossible. So it is difficult to replace the battery. Wireless Sensor Network are prone to node failure due to power loss .The energy efficient shortest path routing algorithm for routing and multi-hop network to communicate every node with sink and have used Ant Colony Optimization to determine new opposition for a sink, so that network communication without any problem which generally occurred due to dead nodes around the sink [4]. These network nodes are having their own environmental and application specific importance. Such as in war zone networks, space network specification, chemical region networks are having the criticalities respective to the network type itself. These networks having the various restrictions in terms of energy, low sensing range, cooperative communication, low power functionality, network size, dense network etc. Due to limited energy, limited hardware resources, low sensing range and critical real time situation increase the failure over the network [6].A considerable amount of energy is consumed to set up a new path whenever it got break. If path break again and again it consumed more energy to setup new path process. It decrease the overall network life time of WSN. .To improve the network life, it is required to generate the optimize route over the network. In this proposed approach, an multi-parameter based hop selection approach is define to generate the optimized route over the network.

In this paper, an multi-parameter based routing approach is defined to generate the effective and reliable route over the network. Working of the proposed routing approach is similar in nature with Distributed Link Quality Aware Routing protocol [1]. . In this work, multi-parameter are considered to generate effective route. It reducing the possible path break and improve the network life time and minimize the energy consumption In this section, the sensor network constraints and issues are explored respective to the routing phenomenon. In section II, the work defined by earlier researchers is discussed. In section III, the proposed methodology is described along with algorithmic approach. In section IV, the results obtained from the work are presented. In section $\mathrm{V}$, the conclusion obtained from the work is presented.

\section{RELATED WORK}

In this section, the work defined by earlier researchers is presented and discussed respective to the routing approaches. M.M. Chandane [1] has defined an Distributed Link Quality Aware Routing approach for optimize route generation over the network. Author define a threshold constraints and link quality parameter to generate the network route. The sensor nodes makes decision of routing based on received link, thus the current node avoid participating in routing ,if the strength of received signal is below the threshold value, thereby reducing the frequency of possible link break and ultimately minimizing the energy consumption. Amandeepsingh, Sunny behal [4] has proposed a new algorithm which is capable of not only to do optimize routing even with that it has the benefit to overcome through pits creating problem around the sink. Authors have used the energy efficient shortest path routing algorithm for routing and multi-hop network to communicate every node with sink and have used Ant Colony Optimization to determine new opposition for a sink, so that network communication without any problem which generally occurred due to dead nodes around the sink. Navjot Sharma, Sunny Behal[6] has defined an proposed algorithm to optimize route distance between nodes and sensors by using Bacteria Foraging optimization algorithm. To prolong the network life time , minimization of energy consumption implemented at all layer of network protocol stack starting from the physical to application layer including cross layer optimization. Hao Wen[8] has defined region aware routing approach in which the storage space is defined to track the region statistics. 
Author defined the node tracking under multiple parameters under history specific analysis. This analysis includes the mobility model analysis and model based route monitoring so that the effective and optimize route was identified. Author defined the node stability analysis to generate the effective route over the network. JiejunKong[9] has defined an ondemand routing approach for effective route generation over the critical network. Author provided the routing solution under two main challenges called route anonymity and back flow criticality analysis. Author defined the location privacy effective routing respective to destination approach. Author defined the optimization to network route under real time scenario. Kyu-Hwan Lee[10] has presented an authentication effective routing approach to optimize the communication approach under security constraints. Author defined an optimization to the network performance and security. The presented work is the analytical observation to the network nodes so that reliable communication over the network will be obtained. AthanasiosBamis[11] has presented a mobility sensitive mobile network optimization to generate the effective route over the network. Author defined the network property analysis under mobile network to analyze these nodes under mobility and speed. Author defined key specific authentication to obtain the reliable next hop so that reliable network communication will be performed. Khaleel Ur Rahman[12] has defined an integrated routing approach to optimize the network communication. Author defined a work on bidirectional infrastructure specific analysis over the mobile network to optimize the network communication and to generate the effective route over the network. Author generated a bridge over the network nodes to select the effective network gateway so that reliable network route will be generated over the network. S.Satish[13] has presented the optimization to the routing approach using ACO approach. This approach is cache oriented and specific to the source node. This routing is energy adaptive and source initiated. The routing is here defined to generate the optimize network path so that reliable network communication will be performed. Giovanni Comarela[14] presented a work to defined robotic routing approach using Ant colony optimization approach. Authorincluded the concept of travelling salesman problem to handle the heuristic state space while generating the network path. Author also proved the effectiveness of work by presenting the comparative analysis with some other approaches. C.DZouza [15] has presented an energy adaptive performance analysis approach to optimize the network communication critical sensor nodes. Author defined a route selection approach using swarm specification. Author also optimize the network deployment and obtain the route dependency to generate the effective route under performance constraints. These constraints includes cost analysis, delay analysis and route length analysis. TaesooJun[16] has presented the optimization to the network route by generating the network strength parameters under multiple constraints. T] hese constraints includes physical characteristics analysis and communication parameter analysis. Author defined the network utility analysis so that the ACO based optimization will be odne. Jing HuiZhong[17 has presented a work to improve the network effectiveness under characteristics analysis so that optimize network route will be generated. Mohsen .Saffarian[18] has defined an ACO specific agent based approach to generate the reliable and secure routing over the network. Author optimized the route over the network using ACO approach. Author reduced the threats over the network and improve the network reliability.

\section{RESEARCH METHOLOGY}

In proposed work, an multi-parameter based hop selection approach is define to generate the optimize route. Sensor node makes decision of routing based on Multi-Parameter.These parameter include energy, failure rate and sensing range. These parameter dynamically analyze the communication route and change the route as per the requirement .Working of the proposed routing approach is similar in nature with Distributed Link Quality Aware Routing protocol. In energy Parameter thus the current node avoids to participating in the routing if energy of node is below the energy threshold value. The current node participating in the routing if energy of node is greater than or equal to threshold value. In failure rate Parameter, thus the current node avoids to participating in the routing if failure rate of node is greater than failure threshold value. Thus current node participating in the routing if failure probability of node is below failure threshold value, thereby minimizing the frequency of possible path break and reduce the energy consumption for path set-up process., ultimately it improve the network life. In distance direction Parameter, if distance from the neighbor node to destination node below the distance from the current node to destination node then set the next effective node as the current node. It means indentify the next effective node in the direction of destination.

In this paper, a multi-parameter specific route generation for sensor network is defined under criticality vector. The work is here about to improve the network life. All the network nodes defined here are homogenous with specification of different energy and criticality vectors. The communication parameters on these nodes are same for all nodes. The work is here proposed to optimize the network route at effective hop selection. The route selection phenomenon defined here includes the node association with network route and other nodes respective to different constraints. The parameters considered in this work include sensing range, energy, failure probability and the directional constraints. In this work, all these parameters are analyzed individually, collectively and cooperatively. Based on this cooperative analysis, the effective next hop selection is done and based on this sequential identification of next hops the route over the network is generated. The work is here defined to perform the effective neighbor selection. The work begin here with the specification of the source and the destination node. The process begin from source node and the neighbor node identification is done at each level under all these parameter till the destination node not occur. This process is repeated till the network path is not generated over the network. The routing algorithm for the work is given in table 1 .

\section{Table 1. Routing Algorithm}

Algorithm(Nodes, N)
/*Nodes is the List of N Nodes defined at random position with
specification of parameters like initial energy, transmission
energy, recieving energy, forwarding enery and failure
probability*/
\{
1. Define Source Node Src and Destination Node Dst
2. $\quad$ Generate Distance Matrix over the network to
identify the distance between each node pair
Energy Effectiveness and Failure Probability




\begin{tabular}{|ll}
\hline 4. & For I=1 to N \\
& {$[$ Perform Node Blockage Analysis on Each } \\
& Node $]$ \\
& \{ \\
5. & if $($ Nodes(I).Energy<EThreshold $)$
\end{tabular}

[A Node having lesser enery is not eligible for communicaiton]

\{

Nodes(I).Block=True;

\}

6. $\quad$ if $(\operatorname{Nodes}(\mathrm{I})$.FailureProb $>$ FThreshold $)$

\{

Nodes(I).Block =True;

\}

\}

7. Set CurNode $=$ Src

[Set Source as Current Node]

8. While CurNode $<>$ Dst

[Repeat Step till Destination Node Not Occur]

\{

9. Set $\mathrm{Nxt}=1$

[Set Nxt as next possible Hop]

10. For $\mathrm{I}=1$ to $\mathrm{N}$

[Find Neighbors to the CurNode] \{

11. if (Dist(CurNode, Nodes(I)) $<$ SensingNode)

[If Node is Neighbor Node]

\{

12. if $(\operatorname{Nodes}(\mathrm{I}) \cdot$ Block=False $)$

[If Node is a valid node]

\{

13. if $\operatorname{Nodes}(\mathrm{i})$.Energy $>\operatorname{Nodes}(\mathrm{Nxt})$.Energy And Dist(Nodes(i), Dst) $<$ Dist(curNode, Dst))

[Identify High Energy Node in direction of destination node]

\{

14. Set $\mathrm{Nxt}=\mathrm{p}$

[Find Next Effective Next Hop]

$$
\text { \} }
$$$$
\text { \} }
$$

15. Set CurNode=Nxt

[Set Next Effective Hop as CurNode]

$$
\}
$$

In this proposed work, an optimization to the routing algorithm is presented for sensor network. The sensor network is a small network of large number tiny sensors distributed at random locations. Each sensor node is defined with some energy. As the communication is performed, each participated node to the communication releases some energy. All these vectors collectively affects the data communication performed over the network. Each associated even gives some energy loss. Because of this there is the requirement of some approach that can reduce the energy consumption over the routing in sensor network. The optimization is here required not only required for energy but also on different parameters. These parameters includes

- Residual energy

- Failure rate

- $\quad$ Sensing range

The optimization is here required to identify the effective route over the network. To identify the effective route, it is required perform the analysis on all possible path. These parameter dynamic analyze the network path it change the path as per reqirement. The presented algorithmic approach has provided the effective route generation so that improve the network life time in terms of dead node, alive node, hop count .

\section{EXPERIMENTAL SETUP}

The simulation is done in matlab environment with specification of following network parameter shown in table 2 .

Table 2 .Simulation Parameters

\begin{tabular}{|c|c|}
\hline Parameter & Value \\
\hline Area & $100 \times 100$ \\
\hline Number of Nodes & 100 \\
\hline Number of Rounds & 100 \\
\hline Initial Energy & Random \\
\hline Transmission Loss & $50 \mathrm{~nJ}$ \\
\hline Receiving Loss & $50 \mathrm{~nJ}$ \\
\hline Forwarding Loss & $10 \mathrm{~nJ}$ \\
\hline Sensing Range & $20 \mathrm{Meter}$ \\
\hline
\end{tabular}

As shown in the table, the work is presented in a generalized network scenario with specification of sensor network constraints. These constraints include the specification of energy consumption constraints over the network so that network communication over the network will be performed.

\section{RESULTS}

The work is here analyzed under different parameters. The results obtained from this analysis is shown here under

Here figure 1 is showing the analysis of dead node over the network in case of existing and proposed work. The comunication is performed for 100 rounds and the analysis of dead nodes is done here. Figure shows that, initially there is no dead node. But as the communication performed, the nodes 
start losing its energy. Till the end of 100 rounds, there are about 28 dead nodes in case of existing approach and 18 nodes are dead in case of proposed approach.

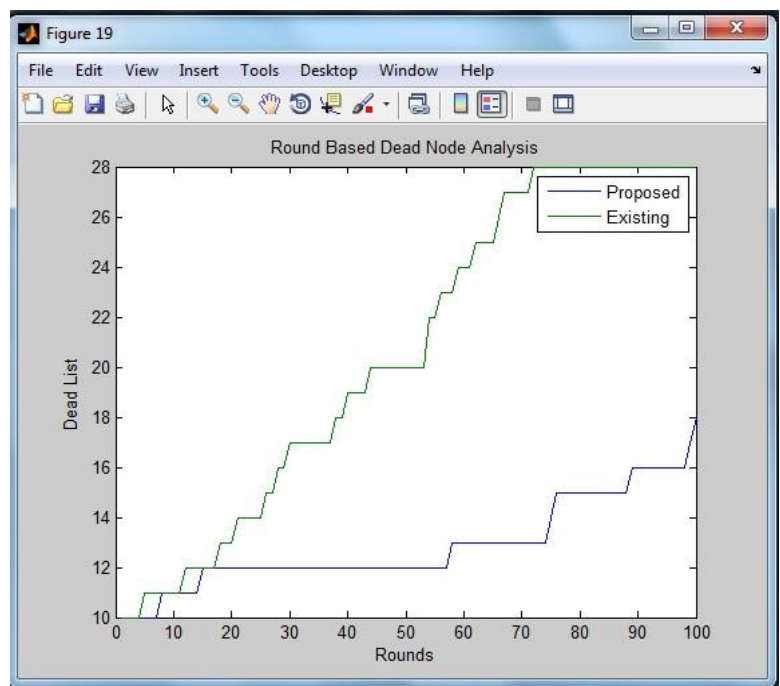

Figure 1 : Dead Node Analysis (Existing Vs. Proposed)

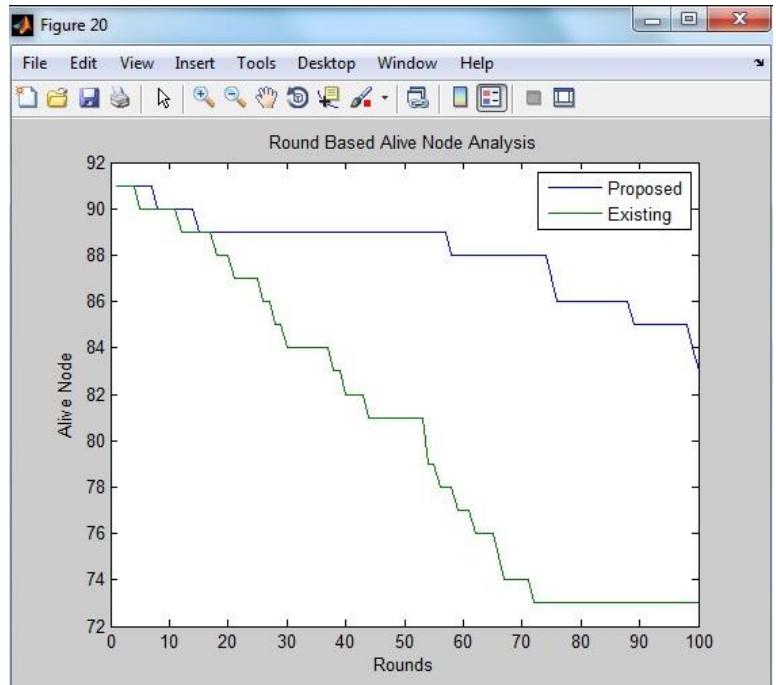

Figure 2 : Alive Node Analysis (Existing Vs. Proposed)

Here figure 2 is showing the analysis of alive node over the network in case of existing and proposed work. As shown in figure, $\mathrm{X}$ axis repersent the number of rounds and $\mathrm{Y}$ axis repersent the alive node over the network..Figure shows that, initially all node are alive. But as the communication performed, the nodes start losing its energy. Till the end of 100 rounds, there are about 73 nodes left alive in case of existing approach and 83 nodes are alive in case of proposed approach.

Here figure 3 is showing the analysis of residual energy over the network in case of existing and proposed approach. As shown in the figure, $X$ axis represents the number of rounds and $y$ axis represents the residual energy over the network. As we can see, the residual energy in case of proposed approach is higher than existing approach.

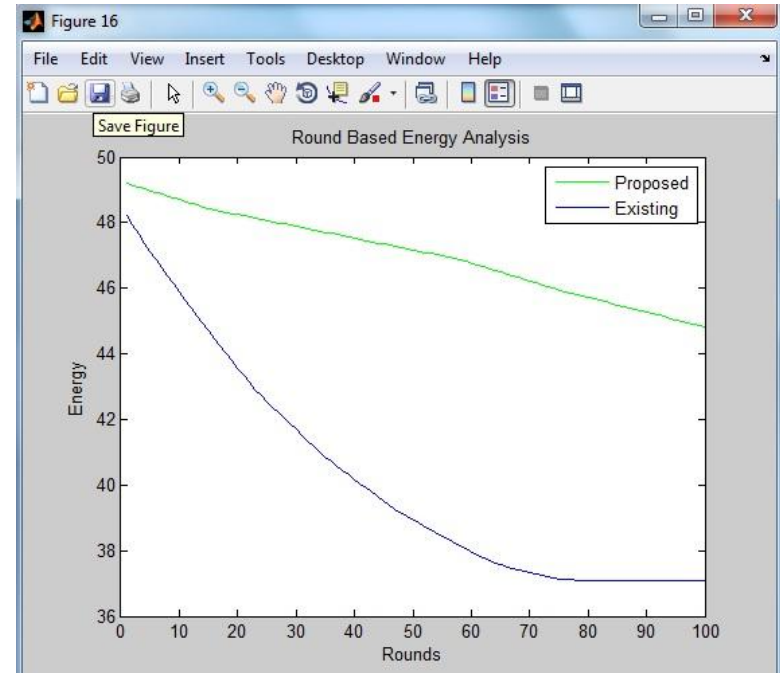

Figure3. Residual Energy Analysis(Existing Vs. Proposed)

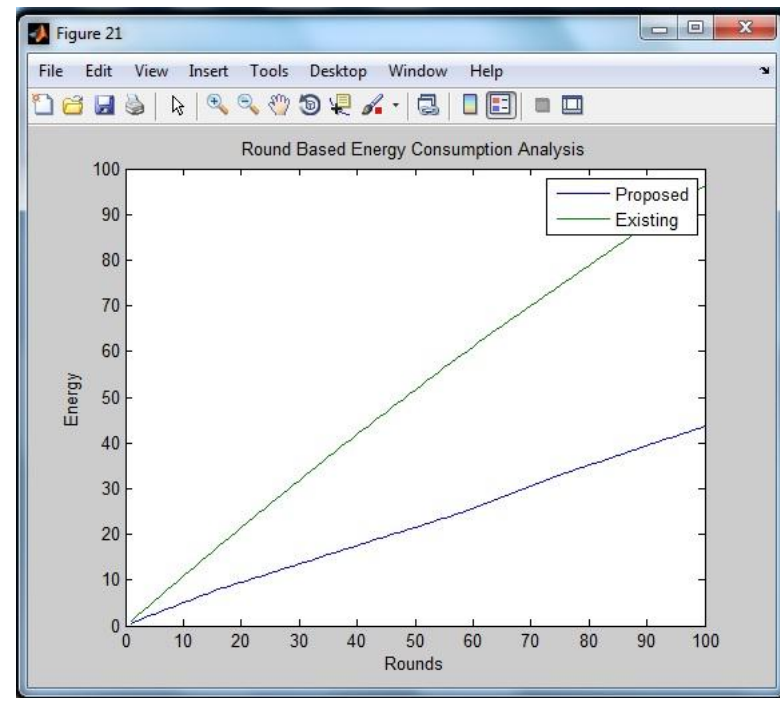

Figure 4. Energy Consumption Analysis(Existing Vs. Proposed)

Here figure 4 is showing the analysis of energy consumption over the network in case of existing and proposed approach. As shown in the figure, $X$ axis represents the number of rounds and $y$ axis represents the energy consumption over the network. As we can see, the energy consumption in case of existing approach is higher than proposed approach.

\section{CONCLUSION}

In this paper,a multi-parameter based hop selection algorithm is proposed to generate the optimized route over the wireless sensor network. The route is here generated under multiple parameters including Residual energy, Failure rate, Sensing Range etc. In this paper, use the threshold constraints to elimination the critical node. It minimize the frequency of possible path break and reduce the energy consumption for path set up process. These parameters are dynamically analyze the communication roué and change the route as per requirement .The number of alive nodes, dead nodes, energy consumption and residual energy etc are computed and analyzed in this proposed work. The obtained results shows that the proposed work has improved the network life in terms dead nodes, alive nodes and reduced the energy consumption 
over the network. The proposed work is defined in normal network behavior but in future some specific consideration can be taken about the network characteristics such as congestion In Proposed work no optimization scheme is implemented but in future some optimization is also be applied to improve the network life time.

\section{REFERENCES}

[1] M..MChandane,"Distributed Link Quality Aware Routung in Wireless Sensor Network:,978-1-4 673-00896/12@2012 IEEE

[2] Vishal Arora, Sunny Behal , Charanjit Singh ," Communication in WSN", $2^{\text {nd }}$ National Conference on challenges \& opportunities in Information Technology(COIT-2008),RIMT-IET, Mar 29-2008,Pg no 202-206.

[3] Amandeep Singh , Sunny Behal," Termite Hill Protocol for network life time in Wireless Sensor Network : Review of Selected Techniques", International journal of Emerging Technology and Advanced Engineering (IJETAE) , Volume 2,Issue 10 ,Page No 453-458, October 2012Tavel, P. 2007 Modeling and Simulation Design. AK Peters Ltd.

[4] AmandeepSingh , Sunny Behal ,"Ant Colony Optimization for improving Network Lifetime in Wireless Sensor Network ", IJAIEM,Vol-2, Issue -4, April 2013, page no 346-352.

[5] Kulvir Singh Sunny Behall, "A Review on Routing Protocols in Wireless Mesh Network", International Journal of Application or Innovation in Engineering \& Management (IJAIEM),pg no. 143-149,Vol-2,Issue-2, Feb 2013

[6] Navjot Sharma, Sunny Behal, "A Systematic way of Soft - Computing Implementation for Wireless Sensor Network Optimization using Bacteria Foraging Optimization Algorithm : A Review", International Journal of Applicaion or Innovation in Engineering \& Management (IJAIEM), Vol-2, Issue-2pg no. 150-154, Feb 2014. [ISSN:2319-4847]

[7] DivyaPrabha, Vishal Kumar Arora," A Survey on LEACH \& its descendent Protocols in WSN ", International Conference on Communication ,Compunting\& System ,2014
[8] Hao Wen," RENA: Region-based Routing in Intermittently Connected Mobile Network", MSWiM'09, October 26-29, 2009, Tenerife, Canary Islands, Spain. ACM 978-1-60558-616-9/09/10.

[9] Jiejun Kong,"ANODR: ANonymous On Demand Routing with Untraceable Routes for Mobile Adhoc Networks", MobiHoc'03, June 1-3, 2003, Annapolis, Maryland, USA ACM 1-58113-684-6/03/0006

[10] Kyu-Hwan Lee," Routing based Authentication for Mobile Ad hoc Network in Home Environment".

[11] AthanasiosBamis," A Mobility Sensitive Approach for Efficient Routing in Ad Hoc Mobile Networks", MSWiM'06, October 2-6, 2006, Torremolinos, Malaga, Spain. ACM 1-59593-477-4/06/0010

[12] Khaleel Ur Rahman Khan," An Efficient Integrated Routing Protocol for Interconnecting Mobile Ad Hoc Networks and the Internet", International Conference on Advances in Computing, Communication and Control (ICAC3'09) ICAC3'09, January 23-24, 2009, Mumbai, Maharashtra, India. ACM 978-1-60558-351-8

[13] S. Sathish," Cache Based Ant Colony Routing Algorithm for Mobile Ad hoc Networks".

[14] Giovanni Comarela," Robot Routing in Sparse Wireless Body Sensor Networks with Continuous Ant Colony Optimization", GECCO'11, July 12-16, 2011, Dublin, Ireland. Copyright 2011 ACM 978-1-4503-0690-4/11/07

[15] C D'Souza," Implementation of Particle Swarm Optimization Based Methodology for Node Placement in Wireless Body Sensor Networks", International Conference and Workshop on Emerging Trends in Technology (ICWET 2011) - TCET, Mumbai, India ICWET'11, February 25-26, 2011, Mumbai, Maharashtra, India. ACM 978-1-4503-0449-8/11/02

[16] Taesoo Jun," Automated Routing Protocol Selection in Mobile Ad Hoc Networks", SAC'07March 1115, 2007, Seoul, Korea ACM 1-59593-480-4/07/0003

[17] Jing-huiZhong," Ant Colony Optimization Algorithm for Lifetime Maximization in Wireless Body Sensor Network with Mobile Sink", GECCO'12, July 7-11, 2012 , Philadelphia, Pennsylvania, USA. ACM 978-1-45031177-9/12/07 